\title{
Digital Artwork
}

\section{Music and Medicine}

Sanjana Sundara Raj Sreenath ${ }^{1}$

\section{Abstract}

\section{Description}

This digital artwork, titled "Music and Medicine", explores the role of holistic patient care as well as the important role of music as a therapeutic tool. Patients often seek connection and belonging during illness, and music serves as a powerful tool in healing. The important role of music in minimizing anxiety and improving mental wellbeing has been brought to the forefront during the Covid pandemic, given the drastic increases in depression and anxiety levels during this time.

Author affiliations are listed at the end of this article.

Correspondence to: Sanjana Sundara Raj Sreenath

Texas Tech University Health Sciences Center 5001 El Paso Dr.

EI Paso, TX 79905

(sanjsund@ttuhsc.edu)

\section{Keywords}

medicine in the arts; humanities; patient care; music; mental healing; holistic care

\section{Conflicts of Interest}

The author declares she has no conflicts of interest.

\section{Author Affiliation}

1. Texas Tech University Health Sciences

Center, EI Paso, TX 


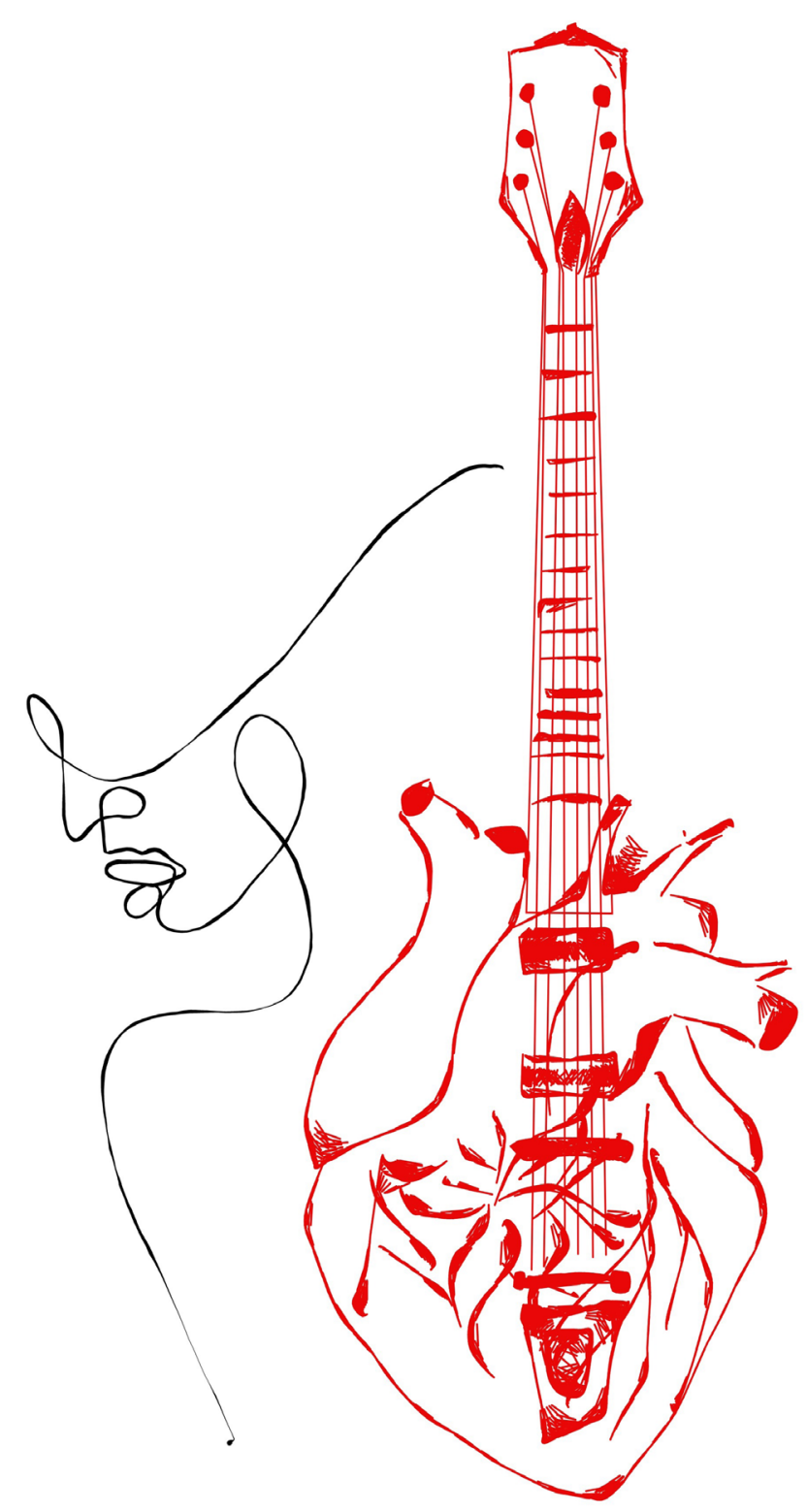

